\title{
Erratum: Apparent rippling with honeycomb symmetry and tunable periodicity observed by scanning tunneling microscopy on suspended graphene [Phys. Rev. B 94, 184302 (2016)]
}

\author{
A. Georgi, P. Nemes-Incze, B. Szafranek, D. Neumaier, V. Geringer, M. Liebmann, and M. Morgenstern \\ (Received 22 November 2016; published 14 December 2016)
}

DOI: 10.1103/PhysRevB.94.219902

Within our paper, we wrongly quoted Ambrosetti et al. [1] with respect to the discussion of errors in the van der Waals interaction between tip and suspended graphene estimated by a sphere with a radius of $R=2 \mathrm{~nm}$ (tip) in distance $d$ above a plane (graphene) using the Hamaker approximation [2],

$$
E_{\mathrm{vdW}, \text { tip }}=\frac{\pi C_{6 A} \rho_{\mathrm{W}} \rho_{\mathrm{G}} R}{6 d},
$$

where parameters to calculate the coefficient $C_{6 A}$ are taken from the literature [3]. A thorough error discussion should read instead: There are a number of possible errors in this estimate. First, the experimentally based Hamaker constants between graphene and $\mathrm{W}$ vary by up to a factor of 2 [3]. Second, the tip radius could be different. It is bound by the fact that we observe atomic resolution, i.e., $R \leqslant 2 \mathrm{~nm}$, but we cannot exclude that the tip is effectively sharper than the tungsten (W) pyramid leading to an effective radius down to $R \simeq 0.8 \mathrm{~nm}$. This reveals another factor of 2.5 of possibly larger van der Waals interactions. Third, the model neglects details of the change in the polarizibility functions by the atomic environment [4]. The adapted $C_{6 A}$ values result, on one hand, from polarizability measurements of a $\mathrm{W}$ tip [3] which should mimic the experimental situation well. On the other hand, the used values for carbon (C) originate from Hartree-Fock calculations of graphite adsorption energies [3] and, thus, might also be close to the experimental ones. Corresponding errors are probably in the range of several $10 \%$. Fourth, screening is neglected, which reduces the van der Waals forces, again in the range of a few 10\% [5]. Fifth, Casimir-Polder forces caused by retardation are neglected, but they start to be of relevance at distances of more than $10 \mathrm{~nm}$ between the interacting materials only $[2,6]$. Finally and most importantly, long-range excitations can lead to an even qualitatively different behavior. The interaction can be enhanced significantly and has been found theoretically to vary by up to a factor of 7 between different $\mathrm{C}$ structures notably exhibiting the largest effective atomic $C_{6 A}$ value for graphene [7]. An increase in atomic $C_{6 A}$ values for C-based molecules with molecular size by up to $20 \%$ is found experimentally, which confirms such a scenario [8]. Density-functional-theory-based calculations even predict that decay exponents are changed due to the long-range excitations, e.g., two graphene layers at about 1 -nm distance exhibit an exponent of -3.5 instead of -4 [1]. Unfortunately, accurate atomic force microscopy measurements of force-distance curves absorb varying exponents in the unknown shape of the tip apex [9]. Thus, without detailed calculations, the resulting error can barely be estimated. However, due to the fact that our parameters are based on experimental values of a W tip and Hartree-Fock calculations of graphite, we are confident that the corresponding error is not too large. So, we expect errors in the van der Waals forces between tip and graphene by up to a factor of 5, which is the largest error in all the estimated forces in our paper.

[1] A. Ambrosetti, N. Ferri, R. A. DiStasio, Jr., and A. Tkatchenko, Science 351, 1171 (2016).

[2] J. N. Israelachvili, Intermolecular and Surface Forces, 2nd ed. (Academic, San Diego, 1992).

[3] A. K. Rappe, C. J. Casewit, K. S. Colwell, W. A. Goddard, and W. M. Skiff, J. Am. Chem. Soc. 114, 10024 (1992); S. C. Wang and T. T. Tsong, Phys. Rev. B 26, 6470 (1982); T. T. Tsong, J. Chem. Phys. 54, 4205 (1971); E. Bichoutskaia and N. C. Pyper, ibid. 128, 024709 (2008).

[4] A. Tkatchenko and M. Scheffler, Phys. Rev. Lett. 102, 073005 (2009).

[5] A. Tkatchenko, R. A. DiStasio, Jr., R. Car, and M. Scheffler, Phys. Rev. Lett. 108, 236402 (2012).
[6] H. B. G. Casimir and D. Polder, Phys. Rev. 73, 360 (1948); U. Hartmann in Scanning Tunneling Microscopy III, 2nd ed., edited by R. Wiesendanger and H. J. Guentherodt (Springer, Berlin, 1996).

[7] V. V. Gobre and A. Tkatchenko, Nat. Commun. 4, 2341 (2013).

[8] C. Wagner, N. Fournier, V. G. Ruiz, C. Li, K. Muellen, M. Rohlfing, A. Tkatchenko, R. Temirov, and F. S. Tautz, Nat. Commun. 5, 5568 (2013).

[9] F. J. Giessibl, Phys. Rev. B 56, 16010 (1997); Rev. Mod. Phys. 75, 949 (2003); H. Hölscher, A. Schwarz, W. Allers, U. D. Schwarz, and R. Wiesendanger, Phys. Rev. B 61, 12678 (2000). 